\section{More is less}

Plants compete for the same resources, such as nutrients, light and water. Because these resources are often limited, the coexistence of plant species requires the creation of trade-offs in resource use. In this issue, Harpole et al. report that increasing a limited nutrient in grassland can eliminate these potential trade-offs, reducing overall species diversity (W. S. Harpole et al. Nature 537, 93-96; 2016).

The authors considered 45 grassland sites across 6 continents, and measured species diversity in response to various nutrient additions. Their results provide strong evidence for a broad ecological theory - that the availability of multiple limiting resources allows plants with different

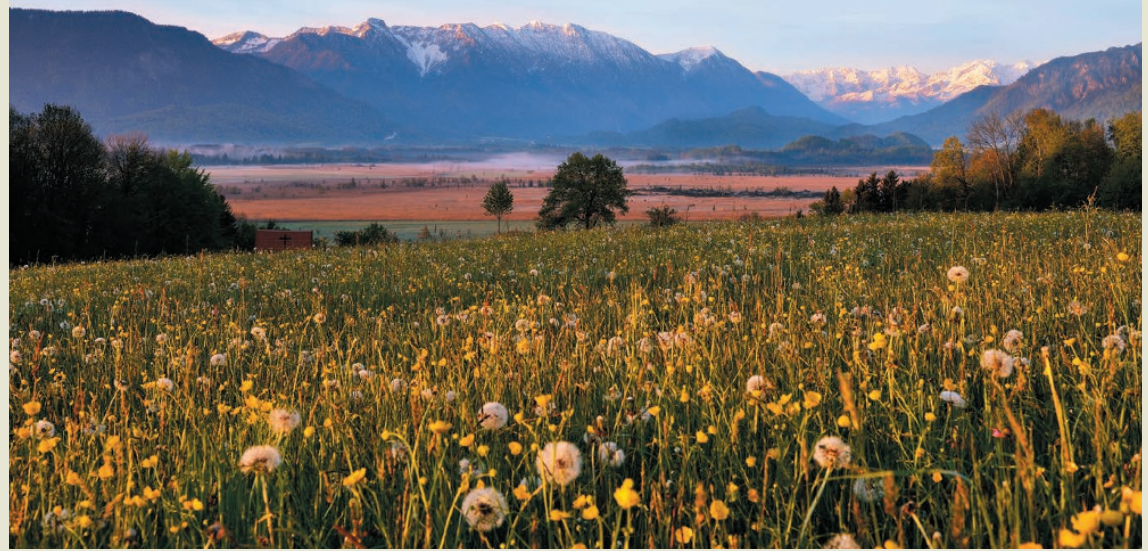

limiting requirements to coexist.

The greater the number of limiting resources that were added, the more species were lost, although productivity and turnover improved.
The researchers argue that, by understanding the mechanisms by which diversity is lost, we might develop strategies for restoring and preserving Earth's biodiversity. Ryan Wilkinson

\title{
CANCER
}

\section{Suffocation of gene expression}

If a tumour outgrows its blood supply, oxygen levels in its cells decrease. It emerges that this change can alter gene expression by limiting the activity of TET enzymes, which remove methyl groups from DNA. SEE ARTICLE P.63

\section{DAN YE \& YUE XIONG}

$\mathrm{T}$ he addition of methyl groups to the DNA base cytosine leads to decreased gene expression, which has broad implications for embryonic development and tumour suppression ${ }^{1}$. Such methylation was once considered to be irreversible, but in 2009 , it was found that ten-eleven translocation (TET) enzymes could catalyse DNA demethylation ${ }^{2}$. This discovery, fuelled by the finding that the gene TET2 is frequently mutated in human blood cancers ${ }^{3}$, sparked intense interest in understanding the function and regulation of this enzyme family. Thienpont et al. ${ }^{4}$ report on page 63 that TET activity is limited by oxygen supply - revealing a general mechanism by which gene expression can be silenced in solid tumours.

TET proteins belong to a dioxygenase enzyme family, members of which depend on three cofactors for their activity: divalent iron $\left(\mathrm{Fe}^{2+}\right)$, the metabolite $\alpha$-ketoglutarate $(\alpha \mathrm{KG})$ and oxygen ${ }^{5} . \mathrm{Fe}^{2+}$ in the active site of the enzyme is coordinated by aKG to split an oxygen molecule into two oxygen atoms. One oxygen atom attacks and

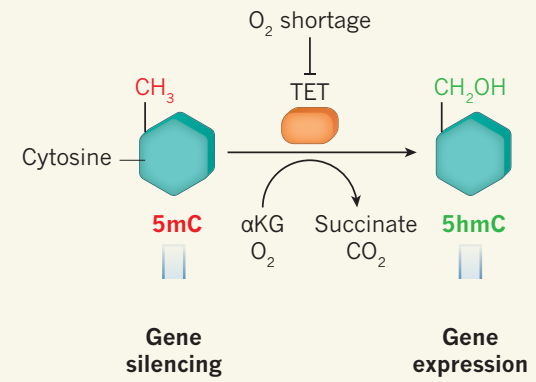

Figure 1 | Reducing TET activity through hypoxia. The addition of a methyl group $\left(\mathrm{CH}_{3}\right)$ to the DNA base cytosine to form 5-methylcytosine $(5 \mathrm{mC})$ can lead to silencing of many genes, including those that suppress tumour development. TET enzymes, acting with the cofactor molecules a-ketoglutarate $(\alpha \mathrm{KG})$ and oxygen, can trigger the demethylation of $5 \mathrm{mC}$. In the first step of this reaction, $\mathrm{O}_{2}$ is split into two atoms. One atom breaks a carbon-carbon bond in $\alpha \mathrm{KG}$, leading to succinate production and carbon dioxide release. The other oxidizes a carbon-hydrogen bond in $\mathrm{CH}_{3}$ to form $\mathrm{CH}_{2} \mathrm{OH}$, converting $5 \mathrm{mC}$ to 5 -hydroxymethylcytosine ( $5 \mathrm{hmC}$ ), eventually leading to gene expression. Thienpont $\mathrm{et} \mathrm{al} .{ }^{4}$ report that a shortage of $\mathrm{O}_{2}$ in solid tumours inhibits TET activity, leading to DNA hypermethylation. breaks a carbon-carbon bond in $\alpha \mathrm{KG}$, leading to the conversion of the metabolite to succinate and the release of carbon dioxide. The other atom oxidizes a carbon-hydrogen bond in the enzyme's substrate (Fig. 1). In TET-mediated reactions, methylated cytosine (5-methylcytosine, $5 \mathrm{mC}$ ) is oxidized to 5 -hydroxymethylcytosine $(5 \mathrm{hmC})$, and further oxidization follows, eventually leading to the removal of methyl groups and so to gene expression ${ }^{6,7}$.

In addition to mutations that inactivate TET genes, TET enzymes can be inactivated in tumours if their cofactors are unavailable. For example, the accumulation of aKG competitors such as the metabolites 2-hydroxyglutarate $(2-\mathrm{HG})^{8,9}$, succinate and fumarate ${ }^{10}$ causes decreased TET activity. The discovery ${ }^{11,12}$ that these three metabolites accumulate in some tumours has led to the idea that cancerpromoting metabolites could have a general role in contributing to tumour development by altering the DNA-methylation landscape in cells, in much the same way that DNA damage causes cancer by altering the genomic landscape. Only a few types of cancer involve mutations in TET genes or show accumulation of aKG-competing metabolites. But the activity of TET enzymes - measured by the production of $5 \mathrm{hmC}$ - seems to be substantially decreased in a wide range of tumours ${ }^{13}$. This discrepancy has remained unexplained until now.

Solid tumours are oxygenated through blood vessels, but a tumour can rapidly outgrow its blood supply, leaving oxygen concentrations low in some regions. Thienpont et al. found that growing human or mouse cancer cells in such hypoxic conditions decreased $5 \mathrm{hmC}$ levels in some, but not all, of the cancer types they examined. Upregulation of TET gene expression 